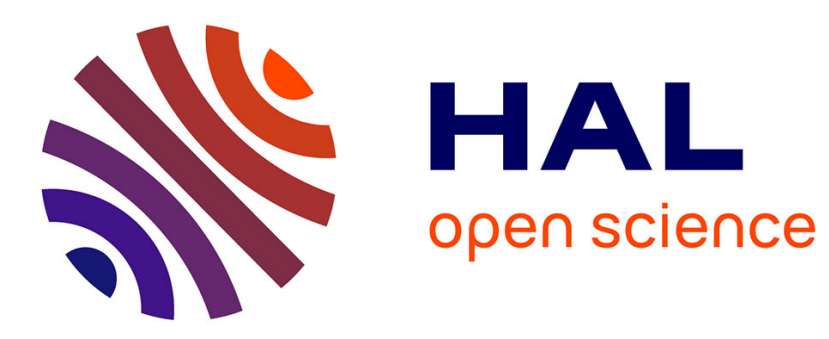

\title{
Nonuniform transformation field analysis of elastic-viscoplastic composites
}

\author{
Sophie Roussette, Jean-Claude Michel, Pierre Suquet
}

\section{To cite this version:}

Sophie Roussette, Jean-Claude Michel, Pierre Suquet. Nonuniform transformation field analysis of elastic-viscoplastic composites. Composites Science and Technology, 2009, 69, pp.22-27. 10.1016/j.compscitech.2007.10.032 . hal-00106307

\section{HAL Id: hal-00106307 https://hal.science/hal-00106307}

Submitted on 1 Dec 2008

HAL is a multi-disciplinary open access archive for the deposit and dissemination of scientific research documents, whether they are published or not. The documents may come from teaching and research institutions in France or abroad, or from public or private research centers.
L'archive ouverte pluridisciplinaire HAL, est destinée au dépôt et à la diffusion de documents scientifiques de niveau recherche, publiés ou non, émanant des établissements d'enseignement et de recherche français ou étrangers, des laboratoires publics ou privés. 


\title{
Nonuniform transformation field analysis of elastic-viscoplastic composites
}

\author{
S. Roussette ${ }^{\mathrm{a}, \mathrm{b}}$, J.C. Michel ${ }^{\mathrm{b}, *}$, P. Suquet ${ }^{\mathrm{b}}$ \\ ${ }^{a}$ CEA Cadarache, LLCC, Bât. 151, 13108 St Paul lez Durance Cedex, France \\ ${ }^{\mathrm{b}}$ Laboratoire de Mécanique et d'Acoustique, CNRS, 31 Chemin Joseph Aiguier, \\ 13402 Marseille Cedex 20, France
}

\begin{abstract}
To exactly describe the macroscopic behaviour of composites with non linear dissipative constituents, it is necessary to take an infinitely large number of internal variables into account. Simplifying assumptions are usually adopted to reduce this number. A new method has been proposed [5], based on the transformation fields approach [1], but not taking transformation fields to be necessarily uniform. The interest of this new method has been shown in the case of composites with elasticplastic constituents. Here we deal with composites having elastic-viscoplastic and porous elastic-viscoplastic constituents. In the latter case, the viscoplastic strain has a dilatational part.
\end{abstract}

Key words: B. Mechanical properties, Modelling, Non-linear behaviour, Plastic deformation, Porosity.

\section{Introduction}

To exactly describe the macroscopic behaviour of composites with non linear dissipative constituents, it is necessary to take an infinitely large number of internal variables into account. Simplifying assumptions are usually adopted to reduce this number. The transformation field analysis (TFA) method initially proposed by [1] for elastic-plastic composites and subsequently extended by other authors (e.g. [2-4]) to composites with more complex behaviour, including damage, provides an elegant means of reducing the number of internal variables by assuming uniform fields of internal variables in individual phases

\footnotetext{
* Corresponding author. Tel: +3349116 4478; fax: +33491164481.
}

Email address: michel@lma.cnrs-mrs.fr (J.C. Michel). 
or sub-domains. Applications of this method to two-phase composites have shown, however, that it is necessary to subdivide the phases into many subdomains to obtain a satisfactory description of the overall behaviour. This subdivision is necessary because of the strong nonlinearity of the constitutive laws involved, which can generate highly heterogeneous mechanical fields within a single phase. In view of these findings, a new method was therefore proposed [5], which consists in decomposing the fields of internal variables into a small number of not necessarily uniform shape functions. Comparisons with the classical transformation field analysis approach have shown the interest of this method in the case of elastic-plastic composite structures [6]. The present study focuses on the case of elastic-viscoplastic and porous elastic-viscoplastic composites. In the latter case, the viscoplastic strain has a dilatational part.

\section{Constitutive relations for the individual constituents}

The constituents on which we focus in the present study are of the elasticviscoplastic and porous elastic-viscoplastic type. These constituents show generalised standard behaviour, defined by the free energy $w$ and the force potential $\psi$. In what follows, only the dissipative mechanisms described by the viscoplastic strain $\varepsilon^{\mathrm{vp}}$ will be taken into account:

$$
w\left(\varepsilon, \varepsilon^{\mathrm{vp}}\right)=\frac{1}{2}\left(\varepsilon-\varepsilon^{\mathrm{vp}}\right): \boldsymbol{L}:\left(\varepsilon-\varepsilon^{\mathrm{vp}}\right), \boldsymbol{\sigma}=\frac{\partial w}{\partial \varepsilon}\left(\varepsilon, \varepsilon^{\mathrm{vp}}\right), \dot{\boldsymbol{\varepsilon}}^{\mathrm{vp}}=\frac{\partial \psi}{\partial \boldsymbol{\sigma}}(\boldsymbol{\sigma}) .
$$

In addition, we will assume the constituents to be isotropic. In this framework, the elastic tensor $\boldsymbol{L}$ is characterised by a bulk modulus $k$ and a shear modulus $G$, and the force potential $\psi$ depends on the stress $\boldsymbol{\sigma}$ only through its second invariant $\sigma_{\text {eq }}$ in the case of classical viscoplastic materials. In the case of porous viscoplastic materials, the force potential also depends on the first invariant $\sigma_{\mathrm{m}}$. Equation (1c) can therefore be written as follows:

$$
\dot{\boldsymbol{\varepsilon}}^{\mathrm{vp}}=\frac{3}{2} \frac{\partial \psi}{\partial \sigma_{\mathrm{eq}}}\left(\sigma_{\mathrm{eq}}, \sigma_{\mathrm{m}}\right) \frac{\boldsymbol{\sigma}^{\mathrm{dev}}}{\sigma_{\mathrm{eq}}}+\frac{1}{3} \frac{\partial \psi}{\partial \sigma_{\mathrm{m}}}\left(\sigma_{\mathrm{eq}}, \sigma_{\mathrm{m}}\right) \boldsymbol{\delta}
$$

where $\boldsymbol{\delta}$ denotes Kronecker's symbol, $\boldsymbol{\sigma}^{\text {dev }}$ the deviatoric part of $\boldsymbol{\sigma}, \sigma_{\mathrm{eq}}=$ $\left(\frac{3}{2} \boldsymbol{\sigma}^{\text {dev }}: \boldsymbol{\sigma}^{\text {dev }}\right)^{1 / 2}$ and $\sigma_{\mathrm{m}}=\operatorname{tr}(\boldsymbol{\sigma}) / 3$. Let us now consider a representative volume element $V$ of a composite material composed of $N$ constituents. The generalised standard structure of the laws in question is known to be preserved in the change of scales. In other words, the composite itself shows generalised 
standard behaviour, but there is an infinitely large number of internal variables involved in its description. The state variables of the system are the macroscopic strain $\boldsymbol{E}$ and the viscoplastic strains $\boldsymbol{\varepsilon}^{\mathrm{vp}}(\boldsymbol{x})$ occurring at all the points $\boldsymbol{x}$ of $V$. Because of the linearity of the local problem (under prescribed state variables), the strain field $\boldsymbol{\varepsilon}(\boldsymbol{x})$ can be decomposed into the following sum:

$$
\boldsymbol{\varepsilon}(\boldsymbol{x})=\boldsymbol{A}(\boldsymbol{x}): \boldsymbol{E}+\frac{1}{|V|} \int_{V} \boldsymbol{D}\left(\boldsymbol{x}, \boldsymbol{x}^{\prime}\right): \boldsymbol{\varepsilon}^{\mathrm{vp}}\left(\boldsymbol{x}^{\prime}\right) d \boldsymbol{x}^{\prime}=\boldsymbol{A}(\boldsymbol{x}): \boldsymbol{E}+\boldsymbol{D} * \boldsymbol{\varepsilon}^{\mathrm{vp}}(\boldsymbol{x}),
$$

where $\boldsymbol{A}(\boldsymbol{x})$ is the elastic strain-localisation tensor and $\boldsymbol{D}\left(\boldsymbol{x}, \boldsymbol{x}^{\prime}\right)$ the non local operator giving the strain at point $\boldsymbol{x}$ resulting from a transformation strain $\boldsymbol{\varepsilon}^{\mathrm{vp}}\left(\boldsymbol{x}^{\prime}\right)$ at point $\boldsymbol{x}^{\prime}$ when the average strain is zero.

\section{Incompressible viscoplasticity}

\subsection{Non uniform transformation fields}

To reduce the number of internal variables and improve the classical transformation field analysis, Michel and Suquet [5] have described a method focusing on the non uniformity of the local fields of internal variables, taking the transformation fields to be non uniform. The viscoplastic strain field is decomposed into a set of fields called plastic modes, $\boldsymbol{\mu}^{k}$ :

$$
\boldsymbol{\varepsilon}^{\mathrm{vp}}(\boldsymbol{x}, t)=\sum_{k=1}^{M} \varepsilon_{k}^{\mathrm{vp}}(t) \boldsymbol{\mu}^{k}(\boldsymbol{x})
$$

By contrast with what occurs with the classical transformation field analysis, the modes $\boldsymbol{\mu}^{k}$ are taken here to be non uniform and tensorial, which means that $\varepsilon_{k}^{\mathrm{vp}}$ is scalar. The total number of modes $M$ can differ from the number $N$ of constituents. Other assumptions are adopted to simplify the theory: (i) The support of each mode is entirely contained in a single phase. (ii) Under incompressible viscoplastic conditions, the modes are traceless tensor fields. (iii) The modes are orthogonal. This prerequisite is met when the modes have their support in different phases, but it has to be imposed when they involve a single phase. (iv) Lastly, to make the reduced internal variables $\varepsilon_{k}^{\mathrm{vp}}$ homogeneous with a strain, the modes are normalised: $\left\langle\mu_{e q}^{k}\right\rangle=1$, where $\mu_{e q}^{k}=\left(\frac{2}{3} \boldsymbol{\mu}^{k}: \boldsymbol{\mu}^{k}\right)^{1 / 2}$. 


\subsection{Reduced variables and influence factors}

Upon multiplying (3) by $\boldsymbol{\mu}^{k}$ and averaging over $V$, given (4), we obtain:

$$
e_{k}=\boldsymbol{a}_{k}: \boldsymbol{E}+\sum_{\ell=1}^{M} D_{k \ell} \varepsilon_{\ell}^{\mathrm{vp}}
$$

where the reduced strain $e_{k}$, the reduced strain-localisation tensor $\boldsymbol{a}_{k}$ and the influence factors $D_{k \ell}$ are defined by:

$$
e_{k}=\left\langle\boldsymbol{\mu}^{k}: \boldsymbol{\varepsilon}\right\rangle, \boldsymbol{a}_{k}=\left\langle\boldsymbol{\mu}^{k}: \boldsymbol{A}\right\rangle, D_{k \ell}=\left\langle\boldsymbol{\mu}^{k}:\left(\boldsymbol{D} * \boldsymbol{\mu}^{\ell}\right)\right\rangle
$$

By analogy with the equation for the reduced strain $e_{k}$ in (6), we define the reduced viscoplastic strain $e_{k}^{\mathrm{vp}}$ and the reduced stress $\tau_{k}$ as follows:

$$
e_{k}^{\mathrm{vp}}=\left\langle\boldsymbol{\mu}^{k}: \boldsymbol{\varepsilon}^{\mathrm{vp}}\right\rangle, \tau_{k}=\left\langle\boldsymbol{\mu}^{k}: \boldsymbol{\sigma}\right\rangle
$$

Note that the whole set of $\varepsilon_{k}^{\mathrm{vp}}$ can be replaced by the whole set of $e_{k}^{\mathrm{vp}}$, since:

$$
e_{k}^{\mathrm{vp}}=\left\langle\boldsymbol{\mu}^{k}: \boldsymbol{\mu}^{k}\right\rangle \varepsilon_{k}^{\mathrm{vp}}
$$

\subsection{Constitutive relations for the reduced variables}

Since the phase elasticity is isotropic and the modes are traceless fields supported in a single phase, the reduced stress $\tau_{k}$ can be written:

$$
\tau_{k}=2 G^{r}\left(e_{k}-e_{k}^{\mathrm{vp}}\right)
$$

where $G^{r}$ is the shear modulus of the phase $r$ involved in mode $k$. The evolution of the reduced viscoplastic strain $e_{k}^{\mathrm{vp}}$ still remains to be described. Using (1c) and the definition (7a) of $e_{k}^{\mathrm{vp}}$, we obtain:

$$
\dot{e}_{k}^{\mathrm{vp}}=\left\langle\boldsymbol{\mu}^{k}: \dot{\boldsymbol{\varepsilon}}^{\mathrm{vp}}\right\rangle=\frac{3}{2}\left\langle\frac{\partial \psi}{\partial \sigma_{\mathrm{eq}}}\left(\sigma_{\mathrm{eq}}\right) \frac{\boldsymbol{\mu}^{k}: \boldsymbol{\sigma}}{\sigma_{\mathrm{eq}}}\right\rangle
$$

At this stage, an approximation is required to obtain a relation between the $\dot{e}_{k}^{\mathrm{vp}}$ 's and the $\tau_{k}$ 's. This is done in [5] by substituting $\tau_{\text {eq }}^{r}=\left[\sum_{k=1}^{M(r)}\left(\tau_{k}\right)^{2}\right]^{1 / 2}$ for $\sigma_{\text {eq }}$ in (10), taking $M(r)$ to denote the number of modes involved in phase $r$ : 


$$
\dot{e}_{k}^{\mathrm{vp}}=\frac{3}{2} \frac{\partial \psi^{r}}{\partial \sigma_{\mathrm{eq}}}\left(\tau_{\mathrm{eq}}^{r}\right) \frac{\tau_{k}}{\tau_{\mathrm{eq}}^{r}}, \tau_{\mathrm{eq}}^{r}=\left[\sum_{k=1}^{M(r)}\left(\tau_{k}\right)^{2}\right]^{1 / 2}
$$

The system of equations (5), (8), (9), (11) provides the set of reduced constitutive relations for the composite. This system is solved along a prescribed loading path, in either the space of macroscopic stresses or that of macroscopic strains [7], where the macroscopic stress is obtained by averaging the stress field which results from (1b), (3) and (4):

$$
\boldsymbol{\Sigma}=\langle\boldsymbol{L}: \boldsymbol{A}\rangle: \boldsymbol{E}+\sum_{k=1}^{M}\left(\left\langle\boldsymbol{L}: \boldsymbol{D} * \boldsymbol{\mu}^{k}\right\rangle-\left\langle\boldsymbol{L}: \boldsymbol{\mu}^{k}\right\rangle\right) \varepsilon_{k}^{\mathrm{vp}}
$$

\subsection{Choosing the modes - the Karhunen-Loève decomposition}

The accuracy of this method depends largely on the modes involved. In the present study, the modes are determined according to the following procedure. Let $\boldsymbol{\theta}^{k}(\boldsymbol{x}), k=1, \ldots, M_{T}(r)$ be the viscoplastic strain fields in a given phase $r$. These fields are determined along certain specific loading paths in the space of macroscopic stresses and at different levels of the macroscopic strain by solving the complete non linear local problem numerically. In all the examples of section 5, a numerical method based on fast Fourier transforms [8,9] was used to determine these viscoplastic strain fields, but any other numerical method as FEM could have been used. The Karhunen-Loève decomposition (also known in the literature as the proper orthogonal decomposition or as the principal component analysis) is then used to build a new set of modes $\boldsymbol{\mu}^{k}(\boldsymbol{x})$, $k=1, \ldots, M_{T}(r)$ :

$$
\boldsymbol{\mu}^{k}(\boldsymbol{x})=\sum_{\ell=1}^{M_{T}(r)} v_{\ell}^{k} \boldsymbol{\theta}^{\ell}(\boldsymbol{x})
$$

where $\boldsymbol{v}^{k}$ are the eigenvectors and $\lambda_{k}$ the eigenvalues of the correlation matrix:

$$
\sum_{j=1}^{M_{T}(r)} g_{i j} v_{j}^{k}=\lambda_{k} v_{i}^{k}, \quad g_{i j}=\left\langle\boldsymbol{\theta}^{i}: \boldsymbol{\theta}^{j}\right\rangle
$$

It can be easily confirmed that the modes $\boldsymbol{\mu}^{k}$ thus obtained are orthogonal: $\left\langle\boldsymbol{\mu}^{k}: \boldsymbol{\mu}^{\ell}\right\rangle=\lambda_{k}$ if $k=\ell$, otherwise 0. If we assume the eigenvalues $\lambda_{k}$ to be arranged in decreasing order of size, another advantage of using the Karhunen-Loève decomposition lies in the fact that only the first $M(r)$ modes 
corresponding to the largest eigenvalues need to be taken into account. In the cases dealt with here, we chose the $M(r)$ modes $\boldsymbol{\mu}^{k}$ making it possible to satisfy the following criterion:

$$
\left(\sum_{k=1}^{M(r)} \lambda_{k}\right) \geq 0.9999\left(\sum_{k=1}^{M_{T}(r)} \lambda_{k}\right)
$$

In practice, this criterion is satisfied with $M(r)=2$ or 3 . As an example, Figure 1 shows the quick decrease in the eigenvalues calculated in the case of a composite with hexagonally distributed fibres, where the fibres and matrix both show power-law elastic-viscoplastic behaviour.

\section{Compressible viscoplasticity}

\subsection{Non uniform transformation fields}

When the viscoplasticity is compressible (as in the case of composites with porous viscoplastic constituents), the viscoplastic strain field can be decomposed as follows:

$$
\boldsymbol{\varepsilon}^{\mathrm{vp}}(\boldsymbol{x}, t)=\sum_{k=1}^{\widetilde{M}} \widetilde{\varepsilon}_{k}^{\mathrm{vp}}(t) \widetilde{\boldsymbol{\mu}}^{k}(\boldsymbol{x})+\sum_{\ell=1}^{\widehat{M}} \widehat{\varepsilon}_{\ell}^{\mathrm{vp}}(t) \widehat{\mu}^{\ell}(\boldsymbol{x}) \boldsymbol{\delta},
$$

where the modes $\tilde{\boldsymbol{\mu}}^{k}$ are traceless tensor fields and the modes $\widehat{\mu}^{\ell}$ are scalar fields. With (16), (3) becomes:

$$
\boldsymbol{\varepsilon}(\boldsymbol{x})=\boldsymbol{A}(\boldsymbol{x}): \boldsymbol{E}+\sum_{k=1}^{\widetilde{M}}\left(\boldsymbol{D} * \widetilde{\boldsymbol{\mu}}^{k}\right)(\boldsymbol{x}) \widetilde{\varepsilon}_{k}^{\mathrm{vp}}+\sum_{\ell=1}^{\widehat{M}}\left(\boldsymbol{D} * \widehat{\mu}^{\ell} \boldsymbol{\delta}\right)(\boldsymbol{x}) \widehat{\varepsilon}_{\ell}^{\mathrm{vp}}
$$

\subsection{Reduced variables and influence factors}

For further purposes, we now introduce the following reduced variables:

$$
\begin{gathered}
\widetilde{e}_{k}=\left\langle\widetilde{\boldsymbol{\mu}}^{k}: \boldsymbol{\varepsilon}\right\rangle, \widetilde{e}_{k}^{\mathrm{vp}}=\left\langle\widetilde{\boldsymbol{\mu}}^{k}: \boldsymbol{\varepsilon}^{\mathrm{vp}}\right\rangle, \widetilde{\tau}_{k}=\left\langle\widetilde{\boldsymbol{\mu}}^{k}: \boldsymbol{\sigma}\right\rangle, \\
\widehat{e}_{k}=\left\langle\widehat{\mu}^{k} \boldsymbol{\delta}: \boldsymbol{\varepsilon}\right\rangle, \widehat{e}_{k}^{\mathrm{vp}}=\left\langle\widehat{\mu}^{k} \boldsymbol{\delta}: \boldsymbol{\varepsilon}^{\mathrm{vp}}\right\rangle, \widehat{\sigma}_{k}=\left\langle\widehat{\mu}^{k} \boldsymbol{\delta}: \boldsymbol{\sigma}\right\rangle .
\end{gathered}
$$


From (17) and the definitions (18a) and (19a) for the reduced strains $\widetilde{e}_{k}$ and $\widehat{e}_{k}$, the following equations can easily be deduced:

$$
\begin{aligned}
& \widetilde{e}_{k}=\widetilde{\boldsymbol{a}}_{k}: \boldsymbol{E}+\sum_{\ell=1}^{\widetilde{M}} \widetilde{D}_{k \ell} \widetilde{\varepsilon}_{\ell}^{\mathrm{vp}}+\sum_{\ell=1}^{\widehat{M}} \widetilde{F}_{k \ell} \widehat{\varepsilon}_{\ell}^{\mathrm{vp}}, \\
& \widehat{e}_{k}=\widehat{\boldsymbol{a}}_{k}: \boldsymbol{E}+\sum_{\ell=1}^{\widetilde{M}} \widehat{F}_{k \ell} \widetilde{\varepsilon}_{\ell}^{\mathrm{vp}}+\sum_{\ell=1}^{\widehat{M}} \widehat{D}_{k \ell} \widehat{\varepsilon}_{\ell}^{\mathrm{vp}},
\end{aligned}
$$

where $\widetilde{\boldsymbol{a}}_{k}=\left\langle\widetilde{\boldsymbol{\mu}}^{k}: \boldsymbol{A}\right\rangle, \widetilde{D}_{k \ell}=\left\langle\widetilde{\boldsymbol{\mu}}^{k}:\left(\boldsymbol{D} * \widetilde{\boldsymbol{\mu}}^{\ell}\right)\right\rangle, \widetilde{F}_{k \ell}=\left\langle\widetilde{\boldsymbol{\mu}}^{k}:\left(\boldsymbol{D} * \widehat{\mu}^{\ell} \boldsymbol{\delta}\right)\right\rangle$, $\widehat{\boldsymbol{a}}_{k}=\left\langle\widehat{\mu}^{k} \boldsymbol{\delta}: \boldsymbol{A}\right\rangle, \widehat{F}_{k \ell}=\left\langle\widehat{\mu}^{k} \boldsymbol{\delta}:\left(\boldsymbol{D} * \widetilde{\boldsymbol{\mu}}^{\ell}\right)\right\rangle$ and $\widehat{D}_{k \ell}=\left\langle\widehat{\mu}^{k} \boldsymbol{\delta}:\left(\boldsymbol{D} * \widehat{\mu}^{\ell} \boldsymbol{\delta}\right)\right\rangle$.

\subsection{Constitutive relations for the reduced variables}

Since the elasticity of the phases is isotropic and the modes supported in a single phase, we have:

$$
\widetilde{\tau}_{k}=2 G^{r}\left(\widetilde{e}_{k}-\widetilde{e}_{k}^{\mathrm{vp}}\right), \quad \widehat{\sigma}_{k}=3 k^{r}\left(\widehat{e}_{k}-\widehat{e}_{k}^{\mathrm{vp}}\right),
$$

where $k^{r}$ and $G^{r}$ denote the bulk and shear moduli of the phase $r$ with which the mode $k$ is associated. Using (2) and the definitions (18b) and (19b) for the reduced viscoplastic strains $\widetilde{e}_{k}^{\mathrm{vp}}$ and $\widehat{e}_{k}^{\mathrm{vp}}$, we obtain:

$$
\dot{\tilde{e}}_{k}^{\mathrm{vp}}=\left\langle\frac{3}{2} \frac{\partial \psi}{\partial \sigma_{\text {eq }}}\left(\sigma_{\text {eq }}, \sigma_{\mathrm{m}}\right) \frac{\tilde{\boldsymbol{\mu}}^{k}: \boldsymbol{\sigma}}{\sigma_{\text {eq }}}\right\rangle, \quad \dot{\hat{e}}_{k}^{\mathrm{vp}}=\left\langle\frac{1}{3} \frac{\partial \psi}{\partial \sigma_{\mathrm{m}}}\left(\sigma_{\text {eq }}, \sigma_{\mathrm{m}}\right) \frac{\widehat{\mu}^{k} \boldsymbol{\delta}: \boldsymbol{\sigma}}{\sigma_{\mathrm{m}}}\right\rangle .
$$

Here again, it is not possible in the non linear context to simply calculate the average of these products, and simplifying assumptions have to be used. In $(23), \sigma_{\text {eq }}$ is replaced by $\widetilde{\tau}_{\text {eq }}^{r}=\left[\sum_{k=1}^{\widetilde{M}(r)}\left(\widetilde{\tau}_{k}\right)^{2}\right]^{1 / 2}$ and $\sigma_{\mathrm{m}}$ is replaced by $\widehat{\sigma}_{\mathrm{m}}^{r}=$ $\left[\sum_{k=1}^{\widehat{M}(r)}\left(\widehat{\sigma}_{k}\right)^{2}\right]^{1 / 2}$, so that the evolution of the reduced internal variables is given by:

$$
\begin{gathered}
\dot{\tilde{e}}_{k}^{\mathrm{vp}}=\frac{3}{2} \frac{\partial \psi^{r}}{\partial \sigma_{\text {eq }}}\left(\widetilde{\tau}_{\text {eq }}^{r}, \widehat{\sigma}_{\mathrm{m}}^{r}\right) \frac{\widetilde{\tau}_{k}}{\widetilde{\tau}_{\text {eq }}^{r}}, \quad \dot{\hat{e}}_{k}^{\mathrm{vp}}=\frac{1}{3} \frac{\partial \psi^{r}}{\partial \sigma_{\mathrm{m}}}\left(\widetilde{\tau}_{\text {eq }}^{r}, \widehat{\sigma}_{\mathrm{m}}^{r}\right) \frac{\widehat{\sigma}_{k}}{\widehat{\sigma}_{\mathrm{m}}^{r}}, \\
\widetilde{\tau}_{\text {eq }}^{r}=\left[\sum_{k=1}^{\widetilde{M}(r)}\left(\widetilde{\tau}_{k}\right)^{2}\right]^{1 / 2}, \quad \widehat{\sigma}_{\mathrm{m}}^{r}=\left[\sum_{k=1}^{\widehat{M}(r)}\left(\widehat{\sigma}_{k}\right)^{2}\right]^{1 / 2},
\end{gathered}
$$


where $\widetilde{M}(r)$ (resp. $\widehat{M}(r)$ ) denotes the number of modes $\widetilde{\boldsymbol{\mu}}^{k}$ (resp. $\widehat{\mu}^{k}$ ) having their support in phase $r$. The macroscopic stress is obtained by averaging the stress field resulting from (1b), (16) and (17):

$$
\begin{gathered}
\boldsymbol{\Sigma}=\langle\boldsymbol{L}: \boldsymbol{A}\rangle: \boldsymbol{E}+\sum_{k=1}^{\widetilde{M}} \widetilde{\boldsymbol{\rho}}_{k} \widetilde{\varepsilon}_{k}^{\mathrm{vp}}+\sum_{k=1}^{\widehat{M}} \widehat{\boldsymbol{\rho}}_{k} \widehat{\varepsilon}_{k}^{\mathrm{vp}}, \\
\widetilde{\boldsymbol{\rho}}_{k}=\left\langle\left(\boldsymbol{L}: \boldsymbol{D} * \widetilde{\boldsymbol{\mu}}^{k}\right)-\left(\boldsymbol{L}: \tilde{\boldsymbol{\mu}}^{k}\right)\right\rangle, \quad \widehat{\boldsymbol{\rho}}_{k}=\left\langle\left(\boldsymbol{L}: \boldsymbol{D} * \widehat{\mu}^{k} \boldsymbol{\delta}\right)-\left(\boldsymbol{L}: \widehat{\mu}^{k} \boldsymbol{\delta}\right)\right\rangle .
\end{gathered}
$$

\subsection{Choosing the modes - the Karhunen-Loève decomposition}

In the case of compressible viscoplastic constituents, the viscoplastic strain fields $\boldsymbol{\theta}^{k}(\boldsymbol{x})$ are decomposed into a purely deviatoric part and a purely spherical part,

$$
\boldsymbol{\theta}^{k}(\boldsymbol{x})=\widetilde{\boldsymbol{\theta}}^{k}(\boldsymbol{x})+\widehat{\theta}^{k}(\boldsymbol{x}) \boldsymbol{\delta}
$$

and the Karhunen-Loève decomposition is applied separately to each of these parts.

\section{Results and discussion}

\subsection{Configurations and material data}

\subsubsection{The case of incompressible viscoplasticity}

The results in this case were obtained on a unidirectional fibre-matrix composite with circular fibres which are regularly distributed at the nodes of a hexagonal array. The volume fraction of the fibres is 0.25 . The fibres and the matrix are assumed to have a power-law elastic-viscoplastic behaviour defined by a force potential of the form:

$$
\psi\left(\sigma_{\mathrm{eq}}\right)=\frac{\sigma_{0} \dot{\varepsilon}_{0}}{n+1}\left(\frac{\sigma_{\mathrm{eq}}}{\sigma_{0}}\right)^{n+1} .
$$

Two different viscosity exponents, corresponding to a linear viscosity and a strongly non-linear viscosity, are considered for the matrix: $n=1$ and $n=8$. The other material properties used with each of these two viscosity exponents are shown in Table 1. 


\subsubsection{The case of compressible viscoplasticity}

The results obtained in this case involved first the same hexagonal configuration as that studied above. The fibres are purely elastic and the matrix is a porous elastic-viscoplastic matrix defined by the elliptical force potential:

$$
\psi\left(\sigma_{\mathrm{eq}}, \sigma_{\mathrm{m}}\right)=\frac{\sigma_{0} \dot{\varepsilon}_{0}}{n+1}\left[\frac{9}{4} A(f)\left(\frac{\sigma_{\mathrm{m}}}{\sigma_{0}}\right)^{2}+B(f)\left(\frac{\sigma_{\mathrm{eq}}}{\sigma_{0}}\right)^{2}\right]^{\frac{n+1}{2}}
$$

where $f$ denotes the porosity and where the expressions of $A(f)$ and $B(f)$ result from [10] starting from an analytical self-consistent scheme and from a bounding method, respectively. Here again, the viscosity exponents $n=1$ and $n=8$ are considered for the matrix. The other material properties used here are shown in Table 2.

In a second step, the results obtained concern a square cell containing a large number of randomly distributed fibres (Figure 4a). The same force potential (28), the same fibre volume fraction (equal to 0.25) and the same material properties (Table 2) are used again here, but only the viscosity exponent $n=1$ is considered for the matrix.

\subsection{Loading}

The composite is subjected to radial load paths in the space of the macroscopic stresses: $\boldsymbol{\Sigma}(t)=\lambda(t) \boldsymbol{\Sigma}_{0}$, where $\boldsymbol{\Sigma}_{0}$ is the prescribed stress direction. The following four stress directions are used:

$$
\begin{array}{ll}
\Sigma_{0}^{(1)}=\boldsymbol{e}_{1} \otimes \boldsymbol{e}_{1}, & \Sigma_{0}^{(2)}=\boldsymbol{e}_{1} \otimes \boldsymbol{e}_{2}+\boldsymbol{e}_{2} \otimes \boldsymbol{e}_{1} \\
\Sigma_{0}^{(3)}=\boldsymbol{e}_{1} \otimes \boldsymbol{e}_{1}-\boldsymbol{e}_{2} \otimes \boldsymbol{e}_{2}, & \Sigma_{0}^{(4)}=\boldsymbol{e}_{1} \otimes \boldsymbol{e}_{1}+\boldsymbol{e}_{2} \otimes \boldsymbol{e}_{2}
\end{array}
$$

\subsection{Discussion}

Figure 2 gives the predictions obtained with various models (TFA, NTFA with one mode per phase calculated at a fixed level of macroscopic strain $\boldsymbol{E}: \boldsymbol{\Sigma}_{0}^{(1)}=5 \%$, and NTFA with two main eigen-modes per phase obtained using the Karhunen-Loève decomposition) when the fibres and the matrix have a power-law elastic-viscoplastic behaviour. The curves correspond to the loading path $\Sigma_{0}^{(1)}$. The predictions obtained with the classical TFA model are too rigid. Those obtained with NTFA model with one mode per phase are satisfactory, but the stress hardening estimate does not match the reference 
calculations. The NTFA model with two main eigen-modes per phase gives excellent results throughout the range of strains under consideration, which shows the usefulness of adopting the Karhunen-Loève decomposition, which means that the information will be optimally distributed at various loading times between the two eigen-modes having the highest eigenvalues.

Figures 3 and $4 \mathrm{~b}$ give the predictions obtained with the NTFA model in which the modes are determined using the Karhunen-Loève decomposition, in the case of elastic fibres and a porous elastic-viscoplastic matrix. The curves in Figures $3 \mathrm{a}$ and $4 \mathrm{~b}$ correspond to the loading paths $\boldsymbol{\Sigma}_{0}^{(3)}$ and $\boldsymbol{\Sigma}_{0}^{(4)}$, whereas those in Figure $3 \mathrm{~b}$ correspond to the loading paths $\boldsymbol{\Sigma}_{0}^{(1)}$ and $\boldsymbol{\Sigma}_{0}^{(2)}$. As in the context of incompressible viscoplasticity, the NTFA method again gives excellent results here.

\section{Conclusion}

The nonuniform transformation fields analysis method was extended here to the case of elastic-viscoplastic and porous elastic-viscoplastic constituents. The predictions obtained were accurate and the number of internal variables involved was reduced thanks to the use of the Karhunen-Loève decomposition.

Acknowledgements. The authors thank Rubens Sampaio for useful discussions about the Karhunen-Loève decomposition.

\section{References}

[1] Dvorak G. Transformation field analysis of inelastic composite materials. Proc. R. Soc. Lond. A 1992;437:311-327.

[2] Kattan P., Voyiadjis G. Overall damage and elastoplastic deformation in fibrous metal matrix composites. Int. J. Plasticity 1993;9:931-949.

[3] Fish J., Shek K. Finite deformation plasticity for composite structures: Computational models and adaptive strategies. Comput. Methods Appl. Mech. Engrg. 1999;172:145-174.

[4] Chaboche J.L., Kruch S., Maire J., Pottier T. Towards a micromechanics based inelastic and damage modeling of composites. Int. J. Plasticity 2001;17:411-439.

[5] Michel J.C., Suquet P. Nonuniform transformation field analysis. Int. J. Solids Struct. 2003;40:6937-6955. 
[6] Michel J.C., Suquet P. Computational analysis of nonlinear composite structures using the nonuniform transformation field analysis. Comput. Methods Appl. Mech. Engrg. 2004;193:5477-5502.

[7] Roussette S. Analyse par champs de transformation de matériaux élastoviscoplastiques multiphases - Application aux combustibles MOX. Doctoral thesis, Université de la Méditerranée (Aix-Marseille 2), France, 2005.

[8] Moulinec H., Suquet P. A numerical method for computing the overall response of nonlinear composites with complex microstructure. Comput. Methods Appl. Mech. Engrg. 1998;157:69-94.

[9] Michel J.C., Moulinec H., Suquet P. Effective properties of composite materials with periodic microstructure: a computational approach. Comput. Methods Appl. Mech. Engrg., 1999;172:109-143.

[10] Michel J.C., Suquet P. The constitutive law of nonlinear viscous and porous materials. J. Mech. Phys. Solids 1992;40:783-812. 
FIGURES 


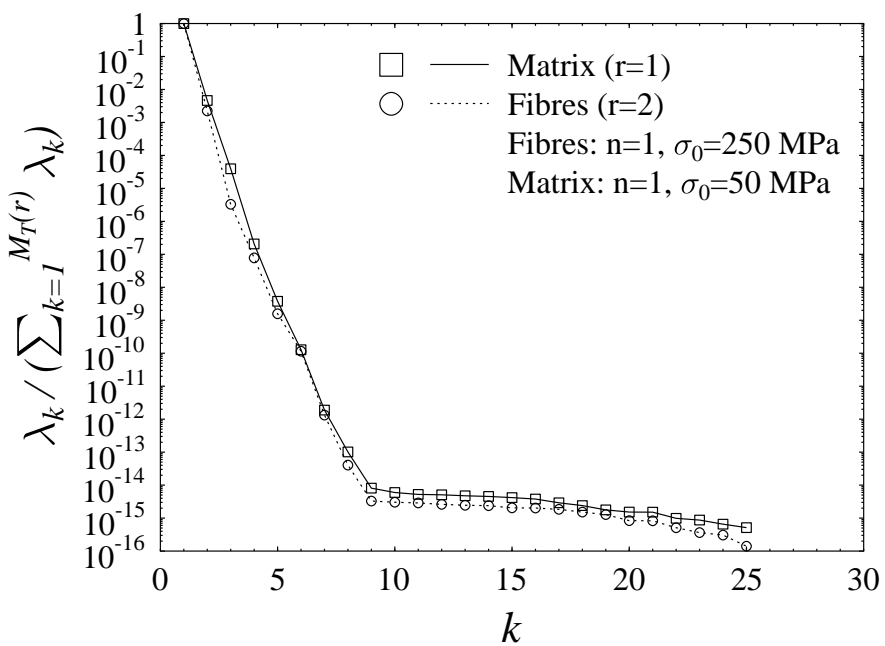

Fig. 1. Example of eigenvalues. 


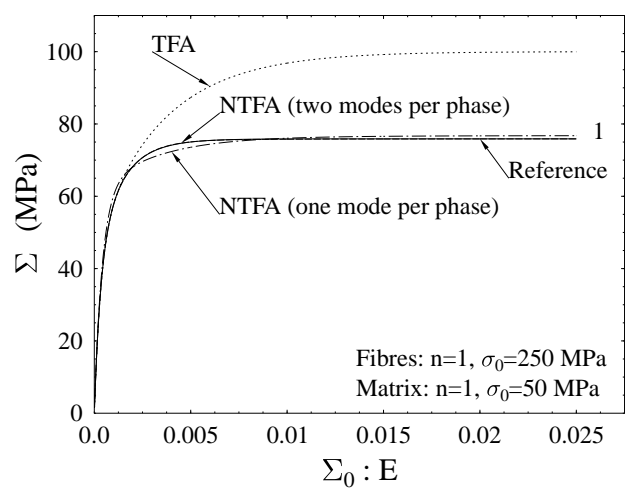

(a)

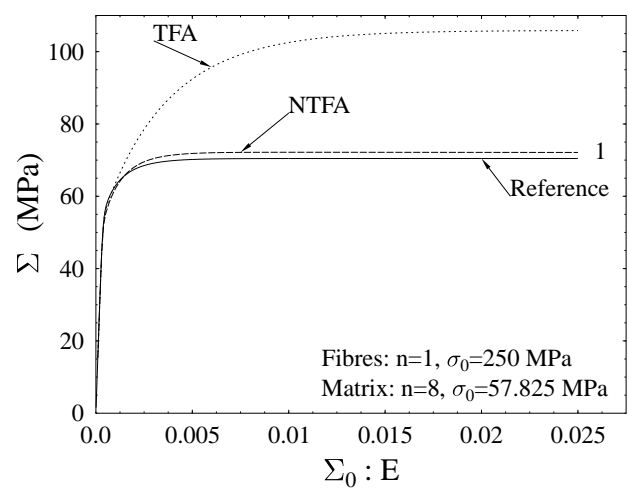

(b)

Fig. 2. Fibres in a hexagonal array. Power-law elastic-viscoplastic fibres and matrix. Loading along $\boldsymbol{\Sigma}_{0}^{(1)}$ (see (29) for the definition of the loading). (a) $n=1$. (b) $n=8$. 


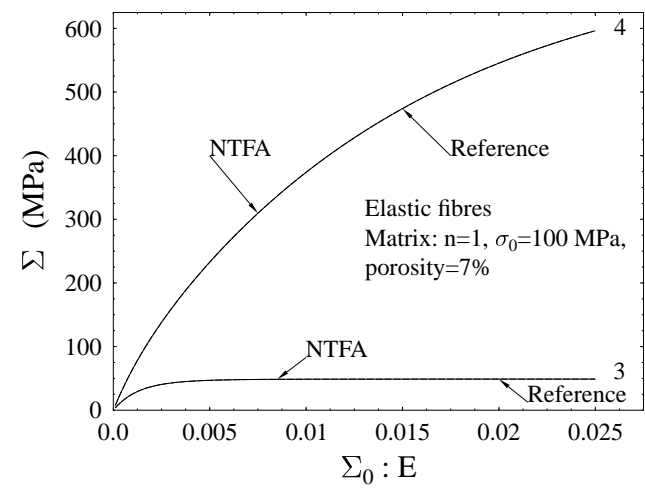

(a)

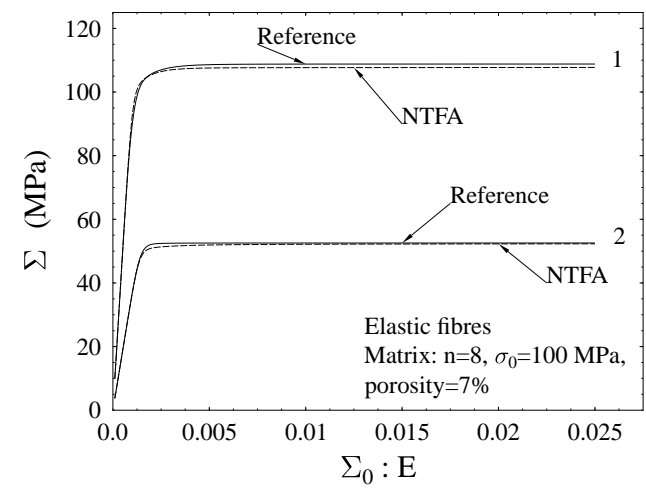

(b)

Fig. 3. Fibres in a hexagonal array. Elastic fibres and porous elastic-viscoplastic matrix. (a) $n=1$. Loading along $\boldsymbol{\Sigma}_{0}^{(3)}$ and $\boldsymbol{\Sigma}_{0}^{(4)}$. (b) $n=8$. Loading along $\boldsymbol{\Sigma}_{0}^{(1)}$ and $\boldsymbol{\Sigma}_{0}^{(2)}$ (see (29) for the definition of the loading). 


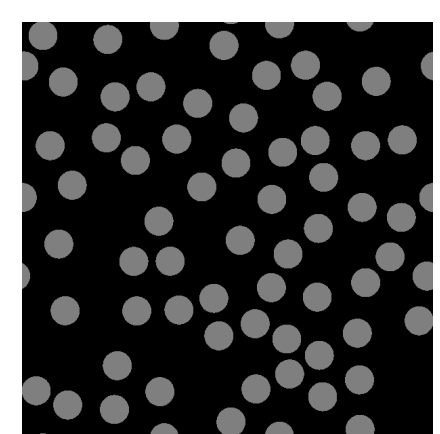

(a)

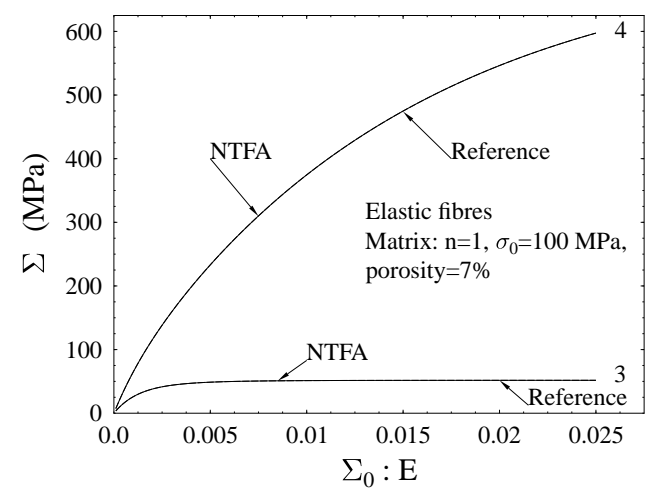

(b)

Fig. 4. Randomly distributed fibres. Elastic fibres and porous elastic-viscoplastic matrix. (a) Microstructure. (b) $n=1$. Loading along $\boldsymbol{\Sigma}_{0}^{(3)}$ and $\boldsymbol{\Sigma}_{0}^{(4)}$ (see (29) for the definition of the loading). 
TABLES 
Table 1

Material properties. Power-law elastic-viscoplastic fibres and matrix.

\begin{tabular}{cccccc} 
& $E(\mathrm{GPa})$ & $\nu$ & $\sigma_{0}(\mathrm{MPa})$ & $\dot{\varepsilon}_{0}\left(s^{-1}\right)$ & $n$ \\
\hline Fibres & 100 & 0.3 & 250 & $10^{-5}$ & 1 \\
\hline Matrix & 180 & 0.3 & 50 & $10^{-5}$ & 1 \\
& 180 & 0.3 & 57.825 & $10^{-5}$ & 8 \\
\hline
\end{tabular}


Table 2

Material properties. Elastic fibres and porous elastic-viscoplastic matrix.

\begin{tabular}{ccccccc} 
& $E(\mathrm{GPa})$ & $\nu$ & $\sigma_{0}(\mathrm{MPa})$ & $\dot{\varepsilon}_{0}\left(s^{-1}\right)$ & $n$ & Porosity \\
\hline Fibres & 400 & 0.2 & & & & \\
\hline Matrix & 70 & 0.3 & 100 & $10^{-2}$ & 1 or 8 & 0.07 \\
\hline
\end{tabular}

